\title{
First-principles study of the phonon dispersion and dielectric properties of wurtzite InP: Role of In $4 d$ electrons
}

\author{
Saikat Mukhopadhyay and Derek A. Stewart* \\ Cornell Nanoscale Facility, 250 Duffield Hall, Cornell University, Ithaca, New York 14853, USA
}

(Received 4 December 2013; revised manuscript received 28 January 2014; published 13 February 2014)

\begin{abstract}
Although wurtzite InP nanowires have recently been grown, an accurate description of the wurtzite InP phonon dispersion is still missing. We calculate the $a b$ initio phonon dispersion of wurtzite and zinc-blende InP using density-functional perturbation theory and a real space supercell approach. Our predicted optical phonon frequencies agree well with measured Raman data from InP nanowires. We find that treating In $4 d$ electrons as valence electrons is required to accurately describe $\mathrm{InP}$ lattice dynamics and dielectric constants, but including spin-orbit coupling has little effect. We also compare the sound velocities and specific heat and find that any difference in the thermal conductivity of InP polytypes should be due to differences in phonon-scattering rates.

DOI: 10.1103/PhysRevB.89.054302

PACS number(s): 63.20.D-, 61.72.uj, 71.15.Mb, 78.20.Ci
\end{abstract}

\section{INTRODUCTION}

Self-assembled nanowires based on III-V materials like InP have shown great promise for a range of applications including field effect transistors [1], photodetectors [2], thermoelectrics [3], and solar cell arrays [4]. This is due in large part to a number of superlative attributes of these systems, including direct band gaps, high mobility electrons, and the ability to selectively dope regions $n$ or $p$ type. Bulk III-V materials, such as arsenides, phosphides, and antimonides, typically have a zinc-blende (ZB) crystal structure. However, the concerted effort to fabricate devices based on III-V nanowires has also shown that it is possible to grow other crystal phases that are unstable in bulk form, such as the wurtzite (WZ), $4 \mathrm{H}$, and $6 \mathrm{H}$ polytypes. This typically occurs due to stacking faults during growth of [111] ZB nanowires. By carefully controlling growth parameters, [0001] WZ nanowires or nanowires with both [111] ZB and [0001] WZ sections can be fabricated.

The ability to modulate the crystal structure during nanowire growth could have important implications for the phonon and thermal properties of nanowires. ZB-WZ interfaces could lead to enhanced phonon scattering and affect the measured Raman signal from these nanowires. In addition, the phonon dispersion along the $\Gamma-A$ line for the WZ phase can be approximately treated as a folding of the phonon branches along the $\Gamma-L$ line in the $\mathrm{ZB}$ phase. This folding leads to additional optical modes at the $\Gamma$ point that could result in additional phonon scattering [5] and reduced thermal conductivity. Currently, only a few works have addressed this issue [5,6], and the lack of accurate phonon-dispersion data on the WZ phases of III-V materials has made progress in this area difficult. A recent work found that WZ InAs nanowires had a lower thermal conductivity than corresponding $\mathrm{ZB}$ nanowires [6]. However, an $a b$ initio analysis of the lattice dynamics of the two phases indicated that the two phases have similar phonon properties (i.e., sound velocity and specific heat), and the difference in the thermal conductivity was attributed to differences in surface scattering. It is interesting to note that, in this case, the $c / a$ ratio for the $\mathrm{WZ}$ phase was

*derek.stewart@cornell.edu predicted to be close to the ideal ratio of $\sqrt{8 / 3}$, indicating that the local environment for atoms would be very similar for the two phases. In the case in which the $c / a$ ratio deviates from the ideal value, the variations in crystal phase could have a strong effect on phonon properties.

While the phonon dispersions of various III-V polytypes have been studied using density-functional theory and other techniques, very few works have considered WZ InP. Available data on the WZ phonon dispersion are currently limited to Raman spectra [7-9] and a fitted rigid-ion model [9]. These works also find different frequencies and symmetries for the WZ Raman peaks. In this work, we will use first-principles approaches to determine the phonon dispersion of $\mathrm{ZB}$ and WZ InP. An accurate description of the phonon dispersion can help confirm previous Raman measurements and identify the symmetry of Raman peaks. In addition, we will also determine the sound velocity and specific heat of WZ InP.

A brief review of previous work on the lattice dynamics of $\mathrm{ZB} \mathrm{InP}$ is necessary to highlight some unresolved issues that could impact our investigation. While an early work [10] using an overlap valence shell model was able to fit the measured ZB InP phonon dispersion [10-12], this empirical approach required ten fitting parameters and did not provide significant physical insight. Ab initio lattice-dynamical calculations for ZB InP [13-15] using density-functional perturbation theory (DFPT) [16] are in excellent agreement for the measured acoustic branches but overestimate the optical frequencies. The match with the experimental phonon dispersion can be improved by increasing the lattice constant slightly [15], but this does not explain the cause of the disagreement. An accurate representation of the optical branches is important for our current investigation given that the only experimental data for WZ InP phonons come from Raman spectroscopy [7-9]. A possible cause for these overestimated optical modes can be found in prior work on the ZB InP dielectric constant. A DFPT study of several III-V semiconductors [13] found that the dielectric constant was overestimated in all cases, except for In-V compounds, (InP, InAs, and InSb), where it was underestimated. The authors suggested that the anomalous behavior in In- $\mathrm{V}$ compounds could be due to the fact that In $4 d$ electrons were not included as valence electrons. However, this hypothesis was not confirmed. While a previous DFPT study [17] found a smaller overestimate of the optical 
TABLE I. Relaxed lattice constants and dielectric constants for ZB and WZ InP shown for QUANTUM ESPRESSO and VASP PAW calculations, experiment, and prior density-functional calculations.

\begin{tabular}{|c|c|c|c|c|c|c|}
\hline & No In $4 d$ & With In $4 d$ & Spin orbit & VASP & Exp. & Prior theory \\
\hline $\mathrm{ZB} a(\AA)$ & 5.7865 & 5.8415 & 5.828 & 5.878 & 5.869 & $5.805[14]$ and $5.9646[22]$ \\
\hline$\epsilon_{\infty}$ & 10.04 & 11.6041 & 11.738 & 12.007 & $9.61(4 \mathrm{~K})[29]$ & $9.04-10.41[13,30-32]$ \\
\hline $\mathrm{WZ} a(\AA)$ & 4.0800 & 4.1215 & 4.1117 & 4.1440 & $4.1423[28]$ & $4.1148[33]$ and 4.1505 [34] \\
\hline$c / a$ & 1.6403 & 1.6432 & 1.6431 & 1.6454 & $1.6419[28]$ & $1.6408[33]$ and $1.6653[34]$ \\
\hline$\epsilon_{\infty}^{x}$ & 9.7152 & 10.917 & 10.987 & 8.956 & & \\
\hline$\epsilon_{\infty}^{z}$ & 9.99 & 11.12 & 11.21 & 10.339 & & \\
\hline
\end{tabular}

frequencies, the authors do not specify if they include In $4 d$ electrons. To resolve this issue, we will perform phonon calculations using In pseudopotentials both with and without $4 d$ electrons. We will also examine the dielectric constants to determine if the $4 d$ electrons improve the comparison with experiment.

Given the recent discovery of $\mathrm{WZ}$ InP nanowires, the lattice dynamics of this phase have been largely unexplored. Gadret et al. examined the optical branches of WZ InP nanowires using a combination of Raman and theoretical calculations [9]. The WZ phonon dispersion in this case was calculated using a 11 parameter rigid-ion model [18] that was fitted to experimental $\mathrm{ZB}$ InP phonon-dispersion data. While this model gives a reasonably good agreement with the measured TO and LO branches at the $\Gamma$ point, it only includes first- and second-nearest neighbor interactions. Since ZB and WZ atomic interactions only deviate for third- and higher-order neighbor interactions, it is unclear if this model will be sufficient to describe the full WZ InP phonon dispersion.

\section{METHODS}

The $a b$ initio phonon dispersions of $\mathrm{ZB}$ and $\mathrm{WZ}$ InP were calculated using DFPT [16] and a real space supercell approach. DFPT calculations were done with the plane-wave pseudopotential code QUANTUM ESPRESSO [19] and used a 80-Ry plane-wave cutoff and the local-density approximation (LDA) for exchange and correlation energies. Previous work found that the local-density approximation does a better job than the generalized gradient approximation (GGA) for III-V systems [20]. For the case in which we neglect In $4 d$ electrons, we used Bachelet, Hamann, and Schlueter (BHS) norm-conserving pseudopotentials [21] with a nonlinear core correction for In. For the case in which In $4 d$ electrons were included, we used ultrasoft pseudopotentials to describe the In and $\mathrm{P}$ ions. Although the spin-orbit splitting of bands is small $(\approx 0.1 \mathrm{eV})$ [22], we examined the effect of spin-orbit coupling on the phonon dispersion using a fully relativistic calculation [23]. A $16 \times 16 \times 16$ Monkhorst-Pack $k$-point grid was used to describe the electronic properties of both crystal structures. For the real space supercell approach, the ground-state electronic structures for the InP polytypes were determined using the plane-wave code VASP $[24,25]$ with the projector augmented wave (PAW) [26] approach, a $8 \times 8 \times 8$ Monkhorst-Pack $k$-point grid, and a $600 \mathrm{eV}$ plane-wave cutoff. To determine the phonon dispersion for $\mathrm{ZB}$ and $\mathrm{WZ}$ InP using DFPT, the dynamical matrices were calculated on a $6 \times 6 \times 6$ Monkhorst-Pack $q$-point grid in the Brillouin zone. Real space phonon calculations were performed with Phonopy [27] on a $2 \times 2 \times 2$ supercell using a $2 \times 2 \times 2 K$-mesh sampling.

Overall, our predicted lattice parameters (Table I) agree well with available theoretical results and experimental data for bulk ZB InP and WZ InP nanowires. Our lattice constants using $4 d$ In electrons for WZ InP $(a=4.1215 \AA, c / a=1.6432$, and $u=0.374289$ ) are close to the measured value in $\mathrm{WZ}$ InP nanowires [28]. For calculations with $4 d$ electrons, we find that the $\mathrm{ZB}$ phase is more stable than the $\mathrm{WZ}$ phase by $0.44 \mathrm{meV} /$ atom. We also did a calculation with normconserving TM FHI pseudopotentials with In $4 d$ electrons and found that the $\mathrm{ZB}$ phase was more stable by $0.43 \mathrm{meV} /$ atom. A few other trends are worth noting. First, the predicted lattice constants using QUANTUM ESPRESSO are all less than the measured lattice constants. It is well known that LDA overbinds, so a small underestimate is expected. However, calculations that neglect $4 d$ electrons have the greatest deviation from experiment, indicating that $4 d$ electrons do play a role in InP bonding. Including spin-orbit coupling and $4 d$ electrons leads to slightly smaller lattice constants than for the case in which only $4 d$ electrons are included. Using VASP PAW, the predicted $\mathrm{ZB}$ InP lattice constant $(5.878 \AA)$ is in good agreement with experiment. For WZ InP, the calculated lattice constant, $4.1440 \AA$, and $c / a$ ratio, 1.6454 , compare well with the measured values, $4.1423 \AA$ and 1.6419 [28], respectively.

\section{PHONON DISPERSIONS}

The ZB InP phonon dispersion calculated using DFPT is shown in Fig. 1(a) for three different pseudopotentials (BHS without In $4 d$ electrons, ultrasoft including In $4 d$ electrons, and ultrasoft with In $4 d$ and spin-orbit coupling). The different pseudopotentials show excellent agreement for the TA branches. While the LA branches predicted using BHS calculations match the other pseudopotentials near the $\Gamma$ point, BHS calculations consistently predict higher phonon frequencies at the Brillouin edges. However, the most significant difference in the calculated phonon dispersion occurs in the optical branches. The BHS optical branches are shifted to higher phonon frequencies, confirming the overestimate observed in previous work [15]. A shift to higher optical frequencies implies stronger bonds and could indicate that neglecting In $4 d$ electrons leads to reduced screening and an overestimate of the bonding strength. This also corresponds well with the smaller lattice constant predicted for this case. Overall, the calculations using spin-orbit coupling only lead to very small differences in the phonon dispersion, at most $1-2 \mathrm{~cm}^{-1}$. Given this close agreement and the computational 

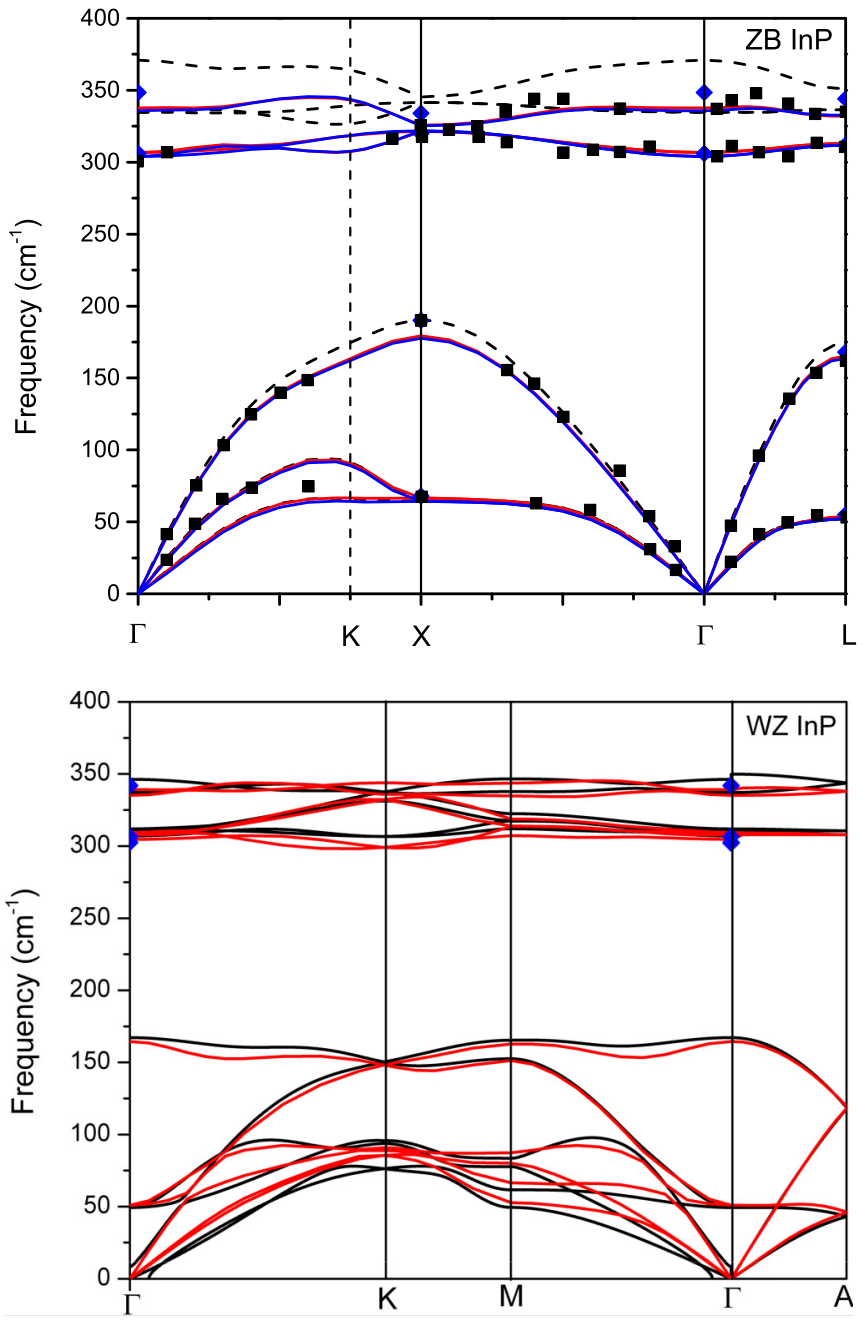

FIG. 1. (Color online) (a) The ZB InP phonon dispersion shown using BHS pseudopotentials without In $4 d$ electrons (black dashed line), ultrasoft pseudopotentials with In $4 d$ electrons (red line), and ultrasoft pseudopotentials with In $4 d$ electrons and spin-orbit interactions (blue line). Neutron-diffraction data [35] (black squares) and Raman measurements [12] (blue diamonds) are shown for comparison. (b) The WZ InP phonon dispersion shown based on PAW (black line), DFPT with $4 d$ electrons (red line), and available Raman data (blue diamonds) [9].

cost involved, we will neglect spin-orbit coupling for the WZ InP phonon-dispersion calculations. Available experimental phonon-dispersion data [12,35] for ZB InP are also shown in Fig. 1(a). The DFPT calculations done with In $4 d$ electrons show excellent agreement with experiment for both acoustic and optical branches, underscoring the importance of including $4 d$ electrons.

Although previous work has shown that GGA significantly overestimates the experimental ZB InP lattice constant [20], it could still potentially provide a better phonon dispersion than the local density approximation. As an additional check, we ran DFPT calculations for ZB InP using GGA pseudopotentials [36,37] both with and without In $4 d$ electrons. We find that GGA does a poor job of reproducing the measured ZB InP optical branches. For example, at the $\Gamma$ point, the predicted TO $\left(279.69 \mathrm{~cm}^{-1}\right)$ and LO $\left(318.92 \mathrm{~cm}^{-1}\right)$ phonon frequencies using GGA without In $4 d$ electrons are considerably lower than the measured values for TO $\left(300.14 \mathrm{~cm}^{-1}\right)$ and LO $\left(349.08 \mathrm{~cm}^{-1}\right)$. Including In $4 d$ electrons slightly improves the TO phonon frequencies $\left(293.94 \mathrm{~cm}^{-1}\right)$, but increases the error in the LO phonon frequency $\left(314.33 \mathrm{~cm}^{-1}\right)$. This indicates that GGA has difficulty in correctly predicting both the lattice constant and phonon dispersion for InP.

We also examined the $\mathrm{ZB}$ InP phonon dispersion using VASP-PAW calculations (see Fig. S1 in supplemental materials [38]). The phonon dispersion obtained from the PAW calculations is consistent with the DFPT calculations and experiments qualitatively. However, PAW calculations slightly underestimate the $\mathrm{TA}_{1}$ branch group velocity compared to the DFPT calculations. DFPT and PAW give similar results for the higher frequency $\mathrm{TA}_{2}$ branch near the Brillouin-zone center, but PAW calculations predict a higher peak frequency along the $\Gamma-X$ direction than both experiment and DFPT calculations. These differences could be due to the fact that the real space supercell calculations take into account fewer nearest-neighbor interactions than the $q$ mesh used in the DFPT calculation. The predicted optical branches using VASP PAW are in much better agreement with experiment than the optical branches predicted using In pseudopotentials without $4 d$ electrons. This is most likely due to the fact that the PAW approach also includes the self-consistent relaxation of semicore states, while the pseudopotential approach only takes into account the $5 \mathrm{~s}$ and $5 p$ In valence states. Previous work on $\mathrm{CaF}_{2}$ also found that PAW was more accurate than pseudopotential calculations that did not take into account the Ca semicore states [39].

The WZ InP phonon dispersion calculated using In $4 d$ electrons and PAW pseudopotentials overall agree well [Fig. 1(b)]. Similar to the ZB case, the PAW group velocity of the TA branches is slightly underestimated compared to DFPT calculations along all symmetry directions except the $\Gamma-L$ direction. The PAW calculations do predict an instability for WZ InP with a small region of negative frequencies near the $\Gamma$ point.

The WZ InP acoustic branches calculated using the rigidion model [9] are significantly different than what we obtained. In their calculation, Gadret et al. [9] found the transverse acoustic branches to be widely separated along several highsymmetry directions (i.e., $\Gamma-K$ ), which indicates reduced symmetry. In our calculations, we find the $\Gamma-K$ TA branches to be nearly identical [Fig. 1(b)]. Our predictions also agree with other In- $V$ WZ crystals (e.g., InAs [6] and InN [40]) where the TA branches along the $\Gamma-K$ line are nearly degenerate. The rigid-ion parameters used in the previous WZ calculations were fit to the $\mathrm{ZB}$ InP phonon dispersion and only include first- and second-nearest neighbor interactions. For optical phonon frequencies at the $\Gamma$ point (the primary focus of Gadret et al.'s paper [9]), this approach is probably sufficient to get reasonable estimates. However, WZ and ZB crystals differ in the third-nearest-neighbor interactions and neglecting these additional terms will make it difficult to accurately predict the full phonon dispersion. This could also lead to the broken symmetries observed in the TA branches along certain symmetry lines. The rigid-ion model was primarily developed for $\mathrm{ZB}$ crystals, and the extension to $\mathrm{WZ}$ is nontrivial. Efforts have been made to scale the localized charge in WZ from its value in the $\mathrm{ZB}$ case. However, the localized charge can 
TABLE II. Calculated optical phonon modes of WZ InP along with modes based on Raman data [9] and a rigid-ion model [9].

\begin{tabular}{lcccc}
\hline \hline & Raman [9] & Rigid model [9] & DFPT & PAW \\
\hline$A_{1}(\mathrm{TO})\left(\mathrm{cm}^{-1}\right)$ & $302.1 \pm 0.8$ & 305.3 & 304.6 & 306.9 \\
$E_{1}(\mathrm{TO})\left(\mathrm{cm}^{-1}\right)$ & $302.4 \pm 0.8$ & 306.3 & 309.2 & 311.8 \\
$E_{2 h}\left(\mathrm{~cm}^{-1}\right)$ & $306.4 \pm 0.7$ & 313.0 & 307.9 & 310.7 \\
$B_{2 h}\left(\mathrm{~cm}^{-1}\right)$ & Not observed & 337.0 & 335.1 & 337.1 \\
$A_{1}(\mathrm{LO})\left(\mathrm{cm}^{-1}\right)$ & $341.9 \pm 0.8$ & 346.4 & 340.2 & 349.8 \\
$E_{1}(\mathrm{LO})\left(\mathrm{cm}^{-1}\right)$ & Not observed & 347.3 & 339.1 & 346.2 \\
\hline \hline
\end{tabular}

depend strongly on other parameters, such as relative ion size in the crystals [41]. Our DFPT calculations, in contrast, take into account force interactions for many nearest neighbors (effectively a $6 \times 6 \times 6 \mathrm{WZ} \mathrm{InP} \mathrm{supercell),} \mathrm{and} \mathrm{our} \mathrm{phonon}$ dispersion is in good agreement with that observed in other WZ structures $[6,40]$.

The calculated optical frequencies are listed in Table II along with measured Raman data and values from the rigid-ion model [9]. The $A_{1}$ (TO) mode $\left(304.6 \mathrm{~cm}^{-1}\right)$ and degenerate $E_{2 h}$ modes $\left(307.9 \mathrm{~cm}^{-1}\right)$ predicted using DFPT are very close to the respective measured Raman values, $302.1 \pm 0.8$ and $306.4 \pm 0.7 \mathrm{~cm}^{-1}$. Similarly, the predicted $A_{1}(\mathrm{LO})$ mode $\left(340.2 \mathrm{~cm}^{-1}\right)$ is within the experimental error for the measured $A_{1}(\mathrm{LO})$ mode $\left(341.9 \mathrm{~cm}^{-1}\right)$. The predicted $B_{2 h}$ frequency is also in good agreement with the rigid-ion model. However, both our calculation using DFPT and the real space approach predict that the $E_{1}(\mathrm{TO})$ mode should have a higher frequency than the $E_{2 h}$ modes. This is in contrast to the Raman analysis and the rigid-ion model, which predict that the $E_{1}(\mathrm{TO})$ mode should be lower than the $E_{2 h}$ modes [9]. A previous work [8] on InP nanowires with mixed $\mathrm{ZB}$ and $\mathrm{WZ}$ sections attributed a Raman peak at $244.7 \mathrm{~cm}^{-1}$ to the $E_{2 h}$ mode. However, based on our predicted phonon dispersion, this peak would be in the phonon band gap that exists between the acoustic and low-lying optical modes and the higher optical branches. It is unclear if this observed mode could arise due to $\mathrm{WZ}-\mathrm{ZB}$ interfaces or surface phonon modes.

In order to provide a more direct comparison with experiment, we also calculated the nonresonant Raman-scattering intensities for WZ InP from density-functional perturbation theory. We followed the approach developed by Porezag and Pederson [42] that has been implemented in the DYNMAT code in the QUANTUM ESPRESSO package. This assumes that the experiment uses a plane-wave polarized laser and that the incident-beam polarization and direction and the observation direction are all orthogonal. As currently implemented, DYNMAT does not work with ultrasoft pseudopotentials, so for these calculations we used norm-conserving FHI LDA pseudopotentials that included In $4 d$ electrons. The predicted optical phonon frequencies show the same trend as our previous results using ultrasoft pseudopotentials, but are shifted to lower frequencies by approximately $3 \mathrm{~cm}^{-1}$ (Fig. 2). Due to the nonanalytic term in the dynamical matrix, the calculated scattering intensities depend on the direction of approach to the $\Gamma$ point. To take this into account, we have performed calculations for $q$ points with either a small $q_{x}$ component or a small $q_{z}$ component. This is equivalent to sampling points along the $\Gamma-M$ and the $\Gamma-A$ lines, respectively. The full data

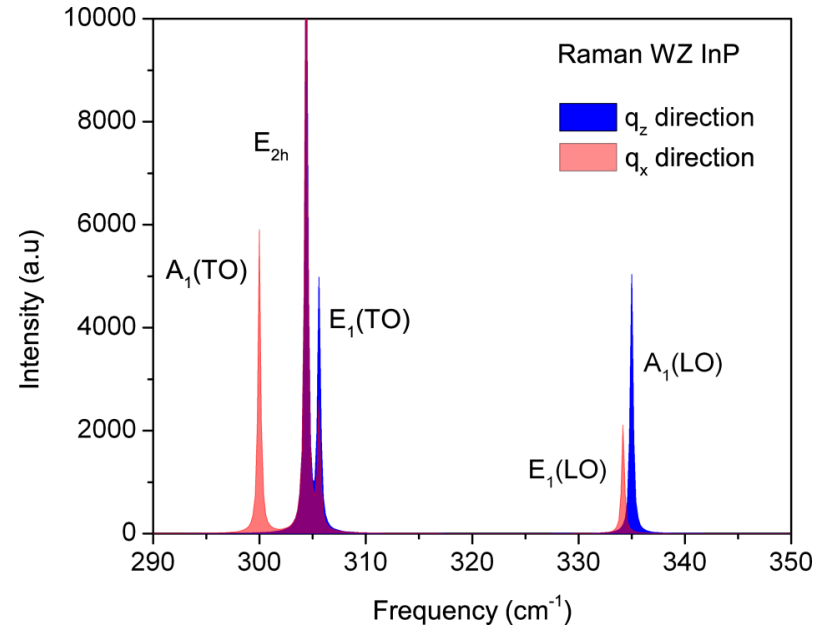

FIG. 2. (Color online) The Raman spectra for $q$ points near the $\Gamma$ point shown in blue and red, respectively, for the case of a small finite $q_{x}$ component along the $\Gamma-M$ symmetry line and for the case of a small finite $q_{z}$ component along the $\Gamma$ - $A$ symmetry line. Regions with overlap between the two cases are shown in purple. A Lorentzian peak shape is assumed with a $1.5 \mathrm{~cm}^{-1}$ full width at half maximum. The symmetry character of the different peaks is also labeled.

for Raman intensities for both $\mathrm{ZB}$ and $\mathrm{WZ}$ InP are included in the supplementary materials (Tables S1 and S2 [38]). Figure 2 shows the predicted Raman spectra in arbitrary units for the two cases assuming a Lorentzian peak shape with a $1.5 \mathrm{~cm}^{-1}$ full width at half maximum.

\section{DIELECTRIC CONSTANTS}

The high-frequency dielectric constants $\left(\epsilon_{\infty}\right)$ of the ZB and WZ forms of InP were also calculated using DFPT (see Table I). Previous studies have found that the predicted dielectric constant can be very sensitive to calculation parameters (e.g., $k$-point mesh and plane-wave cutoff), and we also found that a dense $k$ mesh $(32 \times 32 \times 32)$ was necessary to provide well-converged values. The predicted dielectric constant also depends strongly on the In pseudopotential used. Using a BHS pseudopotential without nonlinear core corrections, the predicted dielectric constant, 9.24, is lower than the measured value (9.61) [29]. In general, previous studies have found that DFPT-LDA predictions overestimate the dielectric constant. An earlier work using BHS pseudopotentials also found a similar anomalous behavior where the dielectric constant was underestimated at 9.04 [13]. Including nonlinear core corrections, our calculated dielectric constant increases to 10.04. This value is close to a previous estimate that included nonlinear core corrections (10.2) [31]. The slight difference is probably due to the lower plane-wave cutoff (24 Ry) used in the earlier work. As noted before, we found that including the In $4 d$ electrons was necessary to provide optical branches in good agreement with experiment. Based on this, we would expect that the most accurate DFPT-LDA estimates for the dielectric constant should also include In $4 d$ electrons. When $4 d$ electrons are included, they can now participate in screening electric fields and the predicted dielectric constant increases to 11.63. If we also include spin-orbit interactions, the dielectric 
constant is slightly higher (11.73), and this corresponds well with the slight reduction in phonon frequencies on adding spin-orbit coupling.

For the case of a WZ crystal, given its lower symmetry, there are two unique terms in the dielectric tensor (in the $a-b$ or basal plane of the crystal, $\epsilon_{\infty}^{x}=\epsilon_{\infty}^{y}$, and in the $c$ direction, $\epsilon_{\infty}^{z}$ ). Regardless of the pseudopotential used, we find the following key trends. First, the dielectric constant in either direction is always less then the predicted value of the zinc-blende case. Second, the dielectric constant in the $z$ direction is always slightly higher than the in-plane value (e.g., for ultrasoft pseudopotentials $\epsilon_{\infty}^{x}=10.91$ and $\left.\epsilon_{\infty}^{z}=11.125\right)$. Previously it has been noted that the dielectric constant varies inversely with the size of the band gap. Although band gaps predicted by DFT are all known to underestimate measured values, our calculated band gaps for $\mathrm{ZB}(0.578 \mathrm{eV})$ and $\mathrm{WZ}$ InP $(0.633 \mathrm{eV})$ do support this trend.

Using the Lyddane-Saches Teller relation [43], we can also calculate the static dielectric constant $\epsilon_{0}$ for the $\mathrm{ZB}$ and $\mathrm{WZ}$ forms of InP. For ZB, this has the simple expression

$$
\frac{\epsilon_{0}}{\epsilon_{\infty}}=\left(\frac{\omega_{\mathrm{LO}}}{\omega_{\mathrm{TO}}}\right)^{2} .
$$

This equation can also be generalized to $\mathrm{WZ}$ and lower symmetry crystals [44]. For the case of WZ crystals, the LO and TO phonon branches with $A_{1}$ and $E_{1}$ symmetry can be used to calculate $\epsilon_{\infty}^{x}$ and $\epsilon_{\infty}^{z}$, respectively [45]. Using the phonon dispersion calculated with ultrasoft pseudopotentials and no spin-orbit coupling, we find for $\mathrm{ZB} \epsilon_{0}=14.076$ and for $\mathrm{WZ} \epsilon_{0}^{x}=13.3$ and $\epsilon_{0}^{z}=13.6$.

Given that ZB and WZ nanowires grow in the [111] and [0001] directions, respectively, we also compared the phonon dispersion along the ZB $\Gamma-L$ line and WZ $\Gamma-A$ line. The WZ $\Gamma-A$ phonon dispersion is typically estimated using a simple folding of the ZB $\Gamma-L$ line. For InP, we find that this works remarkably well (see Fig. 3), and this

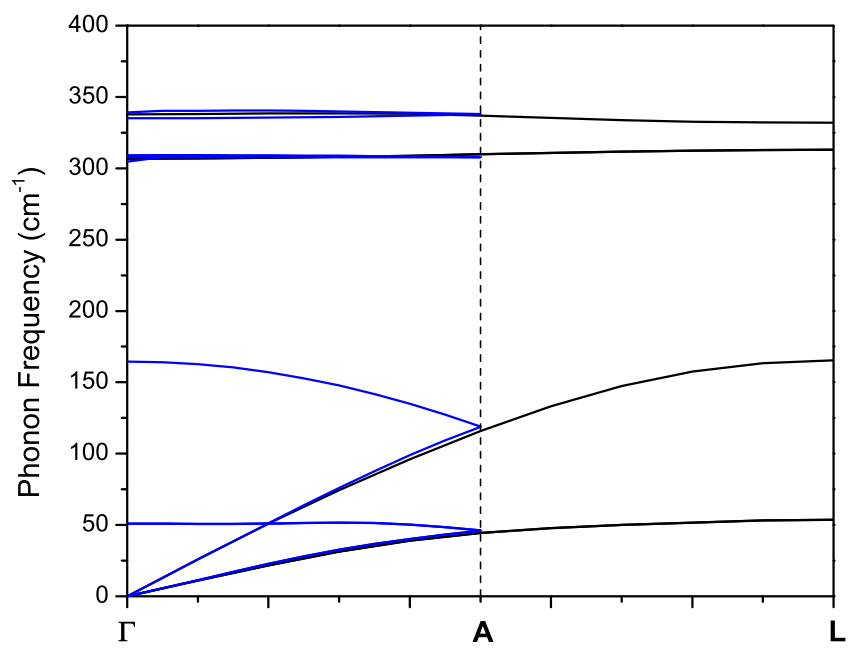

FIG. 3. (Color online) The phonon dispersion for wurtzite InP along the $\Gamma$ - $A$ symmetry line (blue line) shown in comparison with the phonon dispersion for zinc-blende $\mathrm{InP}$ along the $\Gamma-L$ symmetry line (black line). The results are from DFPT calculations using ultrasoft pseudopotentials and including In $4 d$ electrons. also indicates that the LA and TA sound velocities of [0001] WZ and [111] ZB InP nanowires should be nearly identical. Calculated sound velocities in WZ InP along $\Gamma-A, 5.21 \mathrm{~km} / \mathrm{s}$ (LA) and $2.30 \mathrm{~km} / \mathrm{s}$ (TA), are very similar to ZB InP along $\Gamma-L, 5.15$ and $2.20 \mathrm{~km} / \mathrm{s}$. The small difference could be due to the deviation of InP from the ideal wurtzite structure $(c / a=\sqrt{8 / 3})$. Previous work on InAs also found that WZ had a slightly higher sound velocity along the $c$ axis compared to $\mathrm{ZB}$ [6]. However, measured sound velocities in $\mathrm{WZ}$ and $\mathrm{ZB}$ InAs nanowires were found be identical within experimental error [46].

\section{SPECIFIC HEAT AND THERMAL PROPERTIES}

The constant pressure specific heat $\left(C_{p}\right)$ was also calculated for $\mathrm{ZB}$ and $\mathrm{WZ}$ polytypes within the quasiharmonic approximation (Fig. 4). The predicted ZB InP specific heat agrees well with available experimental data [47]. We also find that the WZ InP specific heat is practically identical to that of the $\mathrm{ZB}$ phase. This has also been observed in comparisons of the WZ and ZB phases of InAs [6], GaN [48], and AlN [48].

Although a full thermal conductivity calculation of WZ and $\mathrm{ZB}$ polytypes is beyond the scope of this work, our results do provide some useful information in regard to heat transfer. In a simple kinetic theory picture, thermal conductivity depends on the specific heat (how much energy a phonon can carry), group velocity (how quickly the energy can be transmitted), and phonon-scattering time (interactions that impede heat flow). Our study shows that [111] and [0001] ZB and WZ nanowires should have nearly identical specific heats and group velocities. Therefore, measured differences in the thermal conductivity for these nanowires will most likely be due to differences in scattering rates, possibly due to phonon-phonon interactions [5] or surface scattering [6].

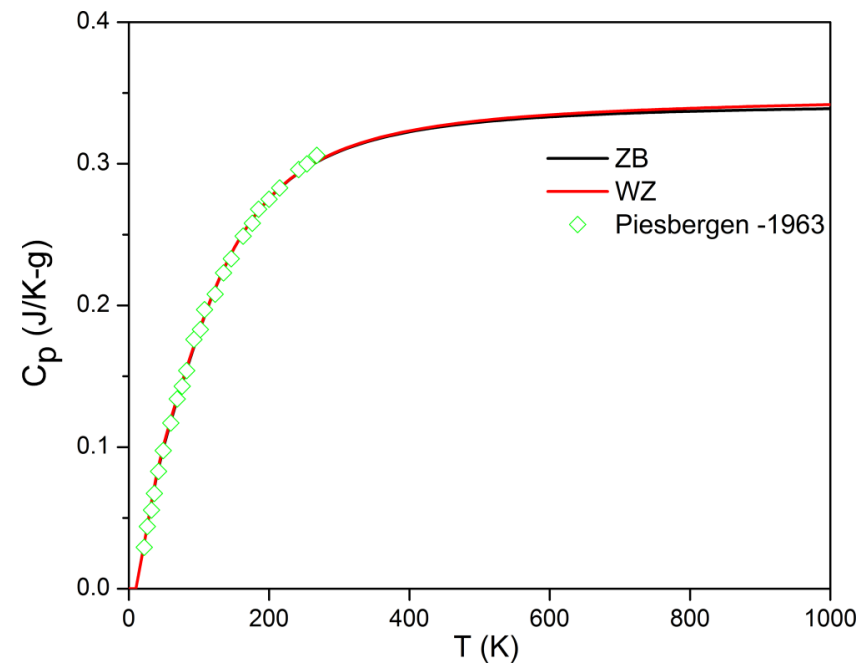

FIG. 4. (Color online) The calculated specific heat at constant pressure as a function of temperature for $\mathrm{ZB}$ (black line) and WZ (red line) InP. The corresponding data of ZB-InP from experiment [47] (open diamonds) are also shown for comparison. 


\section{CONCLUSIONS}

In summary, we have calculated the lattice dynamics of WZ and ZB InP from first principles using DFPT and a real space supercell approach. We find that $4 d$ electrons of In play a critical role in describing the optical branches of InP polytypes. Calculated optical modes show excellent agreement with the experimental Raman data for both $\mathrm{ZB}$ and WZ cases, in which In $4 d$ electrons are included as valence electrons. However, in contrast to some prior work [9], we do find that the $E_{2 h}$ modes should be lower in frequency than the $E_{1}(\mathrm{TO})$ mode. The spin-orbital interaction was also found to have minimal effects on the InP phonon dispersion. Our work provides an accurate WZ InP phonon band structure that is consistent with the general features of other WZ In- $V$ phonon dispersions and that can be compared with future experimental lattice-dynamical studies. For [0001] WZ and [111] InP nanowires, we also confirm that folding the $\mathrm{ZB}$ phonon dispersion along the $\Gamma-L$ symmetry line works quite well as an estimate for the $\Gamma-A$ WZ phonon dispersion. The sound velocity in [0001] WZ InP is also found to agree with that predicted for [111] $\mathrm{ZB}$ InP nanowires, and the specific heats of $\mathrm{ZB}$ and $\mathrm{WZ}$ InP are practically identical. The high frequency and static dielectric constant for WZ InP along the $c$ axis were also shown to be higher than those in the basal plane.

\section{ACKNOWLEDGMENTS}

This research is supported by the NSF under Grant No. 1066406. Calculations were performed at the Cornell Nanoscale Facility, part of the National Nanotechnology Infrastructure Network, funded by the NSF. We also thank Keith Refson for providing access to his Raman spectra script.
[1] K. Tomioka, M. Yoshimura, and T. Fukui, Nature (London) 488 , 189 (2012).

[2] H. Pettersson, I. Zubritskaya, N. T. Nghia, J. Wallentin, M. T. Borgström, K. Storm, L. Landin, P. Wickert, F. Capasso, and L. Samuelson, Nanotechnology 23, 135201 (2012).

[3] P. H. Wu, J. Gooth, X. Zianni, S. F. Svensson, J. G. Gluschke, K. A. Dick, C. Thelander, K. Nielsch, and H. Linke, Nano Lett. 13, 4080 (2013).

[4] J. Wallentin, N. Anttu, D. Asoli, M. Huffman, I. Aberg, M. H. Magnusson, G. Siefer, P. Fuss-Kailuweit, F. Dimroth, B. Witzigmann, H. Q. Xu, L. Samuelson, K. Deppert, and M. T. Borgström, Science 339, 1057 (2013).

[5] A. AlShaikhi and G. P. Srivastava, Phys. Rev. B 76, 195205 (2007).

[6] F. Zhou, A. L. Moore, J. Bolinsson, A. Persson, L. Fröberg, M. T. Pettes, H. Kong, L. Rabenberg, P. Caroff, D. A. Stewart, N. Mingo, K. A. Dick, L. Samuelson, H. Linke, and L. Shi, Phys. Rev. B 83, 205416 (2011).

[7] A. J. Lohn, T. Onishi, and N. P. Kobayashi, Nanotechnology 21, 355702 (2010).

[8] M. Chasnikova, A. Mogilatenko, O. Fedosenko, V. Bryska, A. Petrov, S. Machulik, M. P. Semtsiv, W. Neumann, and W. T. Masselink, J. Cryst. Growth 323, 319 (2011).

[9] E. G. Gadret, M. M. de Lima, J. R. Madureira, T. Chiaramonte, M. A. Cotta, F. Iikawa, and A. Cantarero, Appl. Phys. Lett. 102, 122101 (2013).

[10] P. H. Borcherds and K. Kunc, J. Phys. C 11, 4145 (1978).

[11] G. F. Alfrey and P. H. Borcherds, J. Phys. C 5, L275 (1972).

[12] E. Bedel, G. Landa, R. Carles, J. P. Redoulès, and J. B. Renucci, J. Phys. C 19, 1471 (1986).

[13] S. de Gironcoli, S. Baroni, and R. Resta, Phys. Rev. Lett. 62, 2853 (1989).

[14] J. Fritsch, P. Pavone, and U. Schröder, Phys. Rev. B 52, 11326 (1995).

[15] L. Lindsay, D. A. Broido, and T. L. Reinecke, Phys. Rev. B 87, 165201 (2013).

[16] S. Baroni, S. D. Gironcoli, A. Dal Corso, and P. Giannozzi, Rev. Mod. Phys. 73, 515 (2001).

[17] S. Q. Wang and H. Q. Ye, J. Phys.: Condens. Matter 17, 4475 (2005).
[18] K. Kunc, M. Balkanski, and M. A. Nusimovici, Phys. Rev. B 12, 4346 (1975).

[19] P. Giannozzi et al., J. Phys.: Condens. Matter 21, 395502 (2009).

[20] P. Haas, F. Tran, and P. Blaha, Phys. Rev. B 79, 085104 (2009).

[21] G. B. Bachelet, D. R. Hamann, and M. Schlüter, Phys. Rev. B 26, 4199 (1982).

[22] L. C. O. Dacal and A. Cantarero, Solid State Comm. 151, 781 (2011).

[23] A. Dal Corso, Phys. Rev. B 76, 054308 (2007).

[24] G. Kresse and J. Furthmüller, Comp. Mater. Sci. 6, 15 (1996).

[25] G. Kresse and J. Furthmüller, Phys. Rev. B 54, 11169 (1996).

[26] P. E. Blochl, Phys. Rev. B 50, 17953 (1994).

[27] A. Togo, F. Oba, and I. Tanaka, Phys. Rev. B 78, 134106 (2008).

[28] D. Kriegner, E. Wintersberger, K. Kawaguchi, J. Wallentin, M. T. Borgström, and J. Stangl, Nanotechnology 22, 425704 (2011).

[29] E. T. M. Kernohan, R. T. Phillips, B. H. Bairamov, D. A. Ritchie, and M. Y. Simmons, Solid State Comm. 100, 263 (1996).

[30] F. Kootstra, P. L. de Boeij, and J. G. Snijders, Phys. Rev. B 62, 7071 (2000).

[31] A. Dal Corso, F. Mauri, and A. Rubio, Phys. Rev. B 53, 15638 (1996).

[32] A. DeBernardi, Solid State Comm. 113, 1 (2000).

[33] C. Panse, D. Kriegner, and F. Bechstedt, Phys. Rev. B 84, 075217 (2011).

[34] E. G. Gadret, G. O. Dias, L. C. O. Dacal, M. M. de Lima, C. V. R. S. Ruffo, F. Iikawa, M. J. S. P. Brasil, T. Chiaramonte, M. A. Cotta, L. H. G. Tizei, D. Ugarte, and A. Cantarero, Phys. Rev. B 82, 125327 (2010).

[35] P. H. Borcherds, G. F. Alfrey, D. H. Saunderson, and A. D. B. Woods, J. Phys. C 8, 2022 (1975).

[36] J. P. Perdew, K. Burke, and M. Ernzerhof, Phys. Rev. Lett. 77, 3865 (1996).

[37] C. Hartwigsen, S. Goedecker, and J. Hutter, Phys. Rev. B 58, 3641 (1998).

[38] See Supplemental Material at http://link.aps.org/supplemental/ 10.1103/PhysRevB.89.054302 for technical details. 
[39] N. A. W. Holzwarth, G. E. Matthews, R. B. Dunning, A. R. Tackett, and Y. Zeng, Phys. Rev. B 55, 2005 (1997).

[40] L.-C. Xu, R.-Z. Wang, X. Yang, and H. Yan, J. Appl. Phys. 110, 043528 (2011).

[41] G. Lucovsky, R. M. Martin, and E. Burstein, Phys. Rev. B 4, 1367 (1971).

[42] D. Porezag and M. R. Pederson, Phys. Rev. B 54, 7830 (1996).

[43] R. H. Lydanne, R. G. Sachs, and E. Teller, Phys. Rev. 59, 673 (1941).
[44] W. Cochran and R. A. Cowley, J. Phys. Chem. Solids 23, 447 (1962).

[45] T. Azuhata, T. Sota, K. Suzuki, and S. Nakamura, J. Phys.: Condens. Matter 7, L129 (1995).

[46] S. O. Mariager, D. Khakhulin, H. T. Lemke, K. S. Kjaer, L. Guerin, L. Nuccio, C. B. Sorensen, M. M. Nielsen, and R. Feidenhans, Nano Lett. 10, 2461 (2010).

[47] U. Piesbergen, Z. Naturforsch 18a, 141 (1963).

[48] A. AlShaikhi and G. P. Srivastava, Phys. Status Solidi C 3, 1495 (2006). 